\title{
(WEAK) COMPACTNESS OF HANKEL OPERATORS ON BMOA
}

\author{
Michael PAPADimitrakis
}

\begin{abstract}
We prove that the notions of compactness and weak compactness for a Hankel operator on $B M O A$ are identical.
\end{abstract}

2010 Mathematics Subject Classification: 47B35, 30H35, 30H10.

Key words: Hankel operators, boundedness, compactness, weak compactness, Hardy spaces, bounded mean oscillation, logarithmic bounded mean oscillation.

\section{Introduction and notation}

We write $z \in \mathbb{D}$, where $\mathbb{D}$ is the unit disc in the complex plane, and $\zeta \in \mathbb{T}=\partial \mathbb{D}$, where $\mathbb{T}$ is the unit circle. The usual Lebesgue spaces for $\mathbb{T}$ are denoted by $L^{p}=L^{p}(\mathbb{T})$ and we write $f(\zeta) \sim \sum_{n=-\infty}^{+\infty} \widehat{f}(n) \zeta^{n}$ for the Fourier series of a function $f$ in $L^{1}$. The Hardy spaces for $\mathbb{T}$ are defined by $H^{p}=\left\{f \in L^{p}: \widehat{f}(n)=0\right.$ for $\left.n<0\right\}$. The M. Riesz theorem says that the Riesz projection $P$, defined by

$$
\operatorname{Pf}(\zeta) \sim \sum_{n=0}^{+\infty} \widehat{f}(n) \zeta^{n}
$$

for $f(\zeta) \sim \sum_{n=-\infty}^{+\infty} \widehat{f}(n) \zeta^{n}$, is a bounded operator $L^{p} \rightarrow H^{p}$ when $1<$ $p<\infty$. The Szegö projection or Cauchy transform of $f$ at $z \in \mathbb{D}$ is defined by

$$
\operatorname{Pf}(z)=\frac{1}{2 \pi i} \int_{\mathbb{T}} \frac{f(\zeta)}{\zeta-z} d \zeta
$$

For $1 \leq p<+\infty$ and every $f \in L^{p}$ the $\lim _{r \rightarrow 1-} P f(r \zeta)$ exists for a.e. $\zeta \in \mathbb{T}$ and, when $1<p<+\infty$, this limit is equal to $\operatorname{Pf}(\zeta)$ (where $P$ is the Riesz projection) in both the a.e. sense and in the $L^{p}$ sense. If $p=1$, the $P f(\zeta)=\lim _{r \rightarrow 1-} P f(r \zeta)$ serves as the definition of $P f$, which belongs to the space $L^{1, w}$ of weak- $L^{1}$ functions. In all cases $\operatorname{Pf}(z)$ is an analytic function of $z \in \mathbb{D}$. 
A function $f$ is in $B M O$ if $f \in L^{1}$ and

$$
\|f\|_{*}=\sup _{I} \frac{1}{|I|} \int_{I}\left|f(\zeta)-f_{I}\right||d \zeta|<+\infty,
$$

where $I$ is the general $\operatorname{arc}$ of $\mathbb{T}, f_{I}=\frac{1}{|I|} \int_{I} f(\zeta)|d \zeta|$ and $|I|$ is the length of $I . B M O$ is a Banach space with the norm $\|f\|_{B M O}=|\widehat{f}(0)|+\|f\|_{*}$. The space $B M O A=B M O \cap H^{1}=\{f \in B M O: \widehat{f}(n)=0$ for $n<0\}$ consists of all analytic functions in $B M O$. It is well known that $L^{\infty} \subseteq$ $B M O \subseteq L^{p}$ for $1 \leq p<+\infty$.

The subspace $V M O$ of $B M O$ contains all $f \in L^{1}$ for which

$$
\lim _{|I| \rightarrow 0+} \frac{1}{|I|} \int_{I}\left|f(\zeta)-f_{I}\right||d \zeta|=0
$$

We also define $V M O A=V M O \cap H^{1} . \quad V M O A$ is the closure of analytic polynomials in $B M O A$. The Riesz projection is a bounded operator $L^{\infty} \rightarrow B M O A$ and also $B M O \rightarrow B M O A$.

We then have the spaces $B M O_{\log }$ and $V M O_{\log }$ and their variants $B M O A_{\log }$ and $V M O A_{\log }$. An $f \in L^{1}$ is in $B M O_{\log }$ if

$$
\|f\|_{* *}=\sup _{I} \frac{\log \frac{4 \pi}{|I|}}{|I|} \int_{I}\left|f(\zeta)-f_{I}\right||d \zeta|<+\infty .
$$

$B M O_{\log }$ is a Banach space with the norm $\|f\|_{B M O_{\log }}=|\widehat{f}(0)|+\|f\|_{* *}$. We define $B M O A_{\log }=B M O_{\log } \cap H^{1}$. Clearly, $B M O_{\log } \subseteq B M O$.

The subspace $V M O_{\log }$ of $B M O_{\log }$ contains all $f \in L^{1}$ for which

$$
\lim _{|I| \rightarrow 0+} \frac{\log \frac{4 \pi}{|I|}}{|I|} \int_{I}\left|f(\zeta)-f_{I}\right||d \zeta|=0 .
$$

We also define $V M O A_{\log }=V M O_{\log } \cap H^{1}$.

For each arc $I$ we define $S(I)=\left\{z \in \mathbb{D}: 0<1-|z|<\frac{|I|}{2 \pi}, \frac{z}{|z|} \in I\right\}$, the Carleson square with base $I$. A positive Borel measure $\mu$ in $\mathbb{D}$ is called a Carleson measure if

$$
\sup _{I} \frac{\mu(S(I))}{|I|}<+\infty .
$$

It is known that $\mu$ is a Carleson measure if and only if

$$
\iint_{\mathbb{D}}|f(z)|^{2} d \mu(z) \leq c(\mu) \int_{\mathbb{T}}|f(\zeta)|^{2}|d \zeta|, \quad f \in H^{2}
$$

for some constant $c(\mu)$ and that, if $c(\mu)$ is the smallest such constant,

$$
c(\mu) \asymp \sup _{I} \frac{\mu(S(I))}{|I|},
$$


where $A \asymp B$ means that there are two positive numerical constants $c_{1}$ and $c_{2}$ so that $c_{1} \leq \frac{A}{B} \leq c_{2}$.

We know that $f \in H^{1}$ is in BMOA if and only if the Borel measure $\left|f^{\prime}(z)\right|^{2}\left(1-|z|^{2}\right) d m(z)$ is a Carleson measure and

$$
\|f\|_{*}^{2} \asymp \sup _{I} \frac{1}{|I|} \iint_{S(I)}\left|f^{\prime}(z)\right|^{2}\left(1-|z|^{2}\right) d m(z) .
$$

Similarly, $f \in H^{1}$ is in $V M O A$ if and only if

$$
\lim _{|I| \rightarrow 0+} \frac{1}{|I|} \iint_{S(I)}\left|f^{\prime}(z)\right|^{2}\left(1-|z|^{2}\right) d m(z)=0 .
$$

Analogously, for functions $f$ in $B M O A_{\log }$ we have

$$
\|f\|_{* *}^{2} \asymp \sup _{I} \frac{\log ^{2} \frac{4 \pi}{|I|}}{|I|} \iint_{S(I)}\left|f^{\prime}(z)\right|^{2}\left(1-|z|^{2}\right) d m(z)
$$

and for $f$ in $B M O A_{\log }$

$$
\lim _{|I| \rightarrow 0+} \frac{\log ^{2} \frac{4 \pi}{|I|}}{|I|} \iint_{S(I)}\left|f^{\prime}(z)\right|^{2}\left(1-|z|^{2}\right) d m(z)=0 .
$$

Note that there exists a positive numerical constant $c$ so that $|f(z)| \leq$ $c\|f\|_{B M O} \log \frac{2}{1-|z|^{2}}$ for all $f \in B M O A$ and all $z \in \mathbb{D}$. Conversely, there exists a positive numerical constant $c$ so that for all $z \in \mathbb{D}$ there exists an $f \in B M O A$ with $\|f\|_{B M O}=1$ and $|f(z)| \geq c \log \frac{2}{1-|z|^{2}}$.

Finally, with the Fefferman-Stein duality induced by the binary form

$$
\langle f, g\rangle=\lim _{r \rightarrow 1-} \frac{1}{2 \pi} \int_{\mathbb{T}} f(r \zeta) g(\bar{\zeta})|d \zeta|=\lim _{r \rightarrow 1-} \frac{1}{2 \pi i} \int_{\mathbb{T}} f(r \zeta) g(\bar{\zeta}) \bar{\zeta} d \zeta,
$$

$B M O A$ is isomorphic to $\left(H^{1}\right)^{*}$ and $H^{1}$ is isomorphic to $(V M O A)^{*}$.

Let $a \in H^{2}$ be an analytic symbol with $\widehat{a}(0)=0$. The Hankel operator with symbol $a$ is defined by

$$
H_{a}(f)=P(a J f),
$$

where $J$ is defined by $J f(\zeta)=\bar{\zeta} f(\bar{\zeta}) \sim \sum_{n=-\infty}^{+\infty} \widehat{f}(-n-1) \zeta^{n}$. Note that $J$ turns an analytic function $f$ into an antianalytic function $J f$.

$H_{a}$ is well defined for analytic polynomials $f(\zeta)=\sum_{n=0}^{N} \widehat{f}(n) \zeta^{n}$. The set of analytic polynomials is dense in each $H^{p}(1 \leq p<+\infty)$ and there are classical results which specify, for every particular value of $p$, the necessary and sufficient conditions on the symbol $a$ so that these operators are extended as bounded or even compact operators on $H^{p}$. The situation is described by the following theorems. 
Theorem 1.1 (Nehari, for $p=2$ ). Let $1<p<+\infty$. Then $H_{a}$ is bounded on $H^{p}$ if and only if $a \in B M O A$.

Theorem 1.2 (Hartman, for $p=2$ ). Let $1<p<+\infty$. Then $H_{a}$ is compact on $H^{p}$ if and only if $a \in V M O A$.

Theorem 1.3 (Janson-Peetre-Semmes [3], Cima-Stegenga [2], Tolokonnikov [5]). $H_{a}$ is bounded on $H^{1}$ if and only if $a \in B M O A_{\log }$.

Theorem 1.4 (Papadimitrakis-Virtanen [4]). $H_{a}$ is bounded on $H^{1}$ if and only if $a \in B M O A_{\log }$, in which case

$$
\left\|H_{a}\right\|_{H^{1} \rightarrow H^{1}} \asymp\|a\|_{B M O_{\log }} .
$$

Theorem 1.5 (Papadimitrakis-Virtanen [4]). $H_{a}$ is compact on $H^{1}$ if and only if $a \in V M O A_{\log }$.

Of course, because of the dualities between $H^{1}, B M O A$ and $V M O A$, the above results about $H^{1}$ hold also for BMOA and VMOA.

\section{The main result}

Theorem 2.1. Let $H_{a}$ be bounded on $B M O A$, i.e. $a \in B M O A_{\log }$. Then the following are equivalent:

(1) $H_{a}$ is weakly compact on VMOA.

(2) $H_{a}$ is compact on VMOA.

(3) $H_{a}$ is weakly compact on BMOA.

(4) $H_{a}$ is compact on BMOA.

(5) $H_{a}(B M O A) \subseteq V M O A$.

(6) $a \in V M O A_{\log }$.

There is a similar result in [1] which refers not to the Hankel operator $H_{a}$ but to the Volterra operator

$$
T_{a}(f)(z)=\int_{0}^{z} f(\zeta) a^{\prime}(\zeta) d \zeta .
$$

One might say that, in this respect, the Hankel operator is more difficult than the Volterra operator.

Proof: General considerations show the equivalences between (1), (3) and (5), between (2) and (4) and that (2) implies (1). Also, Theorem 1.5 shows the equivalence between (2) or (4) and (6). Therefore, it remains to prove that (5) implies (6). 
The symbol $a(\zeta)$ of $H_{a}$ and the function $b(z)$ are connected by

$$
b(z)=\frac{1}{2 \pi i} \int_{T} \frac{\bar{\zeta} a(\zeta)}{\zeta-z} d \zeta
$$

or, equivalently, $b(\zeta)=\bar{\zeta} a(\zeta)$ for the boundary values of $b$.

Also, the variable $f(\zeta)$ of $H_{a}(f)$ and $g(z)$ are connected by

$$
g(z)=\frac{1}{2 \pi i} \int_{T} \frac{\overline{f(\bar{\zeta})}}{\zeta-z} d \zeta
$$

or, $g(\zeta)=\overline{f(\bar{\zeta})}$ for the boundary values of $g$.

For any arc $I$ of $\mathbb{T}$ let $z_{I}$ be the midpoint of the inner side of the Carleson square $S(I)$. Let $f \in B M O A$ and $a \in B M O A_{\log }$ or, equivalently, $g \in B M O A$ and $b \in B M O A_{\log }$. Then

$$
\|f\|_{*}=\|g\|_{*}, \quad\|a\|_{* *} \asymp\|b\|_{* *} .
$$

It is easy to show that

$$
H_{a}(f)^{\prime}(z)-\overline{g(z)} b^{\prime}(z)=\frac{1}{2 \pi i} \int_{\mathbb{T}} \frac{(b(\zeta)-b(z) \overline{(g(\zeta)-g(z))}}{(\zeta-z)^{2}} d \zeta
$$

from which we get

$$
\begin{aligned}
\left|H_{a}(f)^{\prime}(z)-\overline{g\left(z_{I}\right)} b^{\prime}(z)\right| \leq & \frac{1}{2 \pi} \int_{\mathbb{T}} \frac{|b(\zeta)-b(z)||g(\zeta)-g(z)|}{|\zeta-z|^{2}}|d \zeta| \\
& +\left|g(z)-g\left(z_{I}\right)\right|\left|b^{\prime}(z)\right| .
\end{aligned}
$$

Applying the Cauchy-Schwarz inequality together with standard estimates for functions in $B M O A$ and in $B M O A_{\log }$, we get

$$
\frac{1}{2 \pi} \int_{\mathbb{T}} \frac{|b(\zeta)-b(z)||g(\zeta)-g(z)|}{|\zeta-z|^{2}}|d \zeta| \leq c\|b\|_{* *}\|g\|_{*} \frac{1}{\left(1-|z|^{2}\right) \log \frac{2}{1-|z|^{2}}} .
$$

In what follows, $c$ denotes a positive numerical constant, not necessarily the same at each occurrence. This, for every arc $I$, implies

$$
\begin{aligned}
\frac{1}{|I|} \iint_{S(I)}\left|\left(H_{a} f\right)^{\prime}(z)-\overline{g\left(z_{I}\right)} b^{\prime}(z)\right|^{2}\left(1-|z|^{2}\right) d m(z) \\
\leq c\|b\|_{* *}^{2}\|g\|_{*}^{2} \frac{1}{|I|} \iint_{S(I)} \frac{1}{\left(1-|z|^{2}\right) \log ^{2} \frac{2}{1-|z|^{2}}} d m(z) \\
\quad+\frac{c}{|I|} \iint_{S(I)}\left|g(z)-g\left(z_{I}\right)\right|^{2}\left|b^{\prime}(z)\right|^{2}\left(1-|z|^{2}\right) d m(z) \\
=A+B
\end{aligned}
$$


A direct calculation of the integral of the term $A$ gives

$$
A \leq c \frac{1}{\log \frac{4 \pi}{|I|}}\|b\|_{* *}^{2}\|g\|_{*}^{2} .
$$

Observing that $\left|1-\overline{z_{I}} z\right| \asymp|I|$ for all $z \in S(I)$ and considering the Borel measure $d \mu(z)$ which is equal to $\left|b^{\prime}(z)\right|^{2}\left(1-|z|^{2}\right) d m(z)$ on $S(I)$ and equal to zero on $\mathbb{D} \backslash S(I)$, we find

$$
\begin{aligned}
B & \leq c|I| \iint_{S(I)} \frac{\left|g(z)-g\left(z_{I}\right)\right|^{2}}{\left|1-\overline{z_{I}} z\right|^{2}}\left|b^{\prime}(z)\right|^{2}\left(1-|z|^{2}\right) d m(z) \\
& \leq c|I| \sup _{J} \frac{\mu(S(J))}{|J|} \int_{\mathbb{T}} \frac{\left|g(\zeta)-g\left(z_{I}\right)\right|^{2}}{\left|1-\overline{z_{I}} \zeta\right|^{2}}|d \zeta| \\
& \leq c \sup _{J} \frac{\mu(S(J))}{|J|} \int_{\mathbb{T}}\left|g(\zeta)-g\left(z_{I}\right)\right|^{2} \frac{1-\left|z_{I}\right|^{2}}{\left|\zeta-z_{I}\right|^{2}}|d \zeta| \\
& \leq c\|g\|_{*}^{2} \sup _{J} \frac{\mu(S(J))}{|J|} .
\end{aligned}
$$

Estimating $\frac{\mu(S(J))}{|J|}=\frac{\mu(S(J) \cap S(I))}{|J|}$, we observe that we need only consider $\operatorname{arcs} J$ with $J \cap I \neq \emptyset$. If $|J|>|I|$, then $\frac{\mu(S(J))}{|J|} \leq \frac{\mu(S(I))}{|I|}$. If $|J| \leq|I|$, then $J \subseteq 3 I$, where $3 I$ is the arc with the same midpoint as $I$ and with length three times the length of $I$. Hence, in both cases we get

$$
\begin{aligned}
\sup _{J} \frac{\mu(S(J))}{|J|} & \leq \sup _{J \subseteq 3 I} \frac{1}{|J|} \iint_{S(J)}\left|b^{\prime}(z)\right|^{2}\left(1-|z|^{2}\right) d m(z) \\
& \leq c \sup _{J \subseteq 3 I} \frac{1}{\log ^{2} \frac{4 \pi}{|J|}}\|b\|_{* *}^{2} \leq \frac{c}{\log ^{2} \frac{4 \pi}{|I|}}\|b\|_{* *}^{2} .
\end{aligned}
$$

Therefore,

$$
B \leq \frac{c}{\log ^{2} \frac{4 \pi}{|I|}}\|b\|_{* *}^{2}\|g\|_{*}^{2}
$$

and, finally,

$$
\frac{1}{|I|} \iint_{S(I)}\left|H_{a}(f)^{\prime}(z)-\overline{g\left(z_{I}\right)} b^{\prime}(z)\right|^{2}\left(1-|z|^{2}\right) d m(z) \leq \frac{c}{\log \frac{4 \pi}{|I|}}\|b\|_{* *}^{2}\|g\|_{*}^{2} .
$$

All the previous estimates of the terms $A$ and $B$ are contained in [4] and played a substantial role in the proofs of Theorems 1.4 and 1.5. We included them here for the sake of completeness. We also changed them slightly to make them more accessible for the present work. 
Although this is not our immediate purpose, it is straightforward to show that (6) implies (5). Suppose that $b \in V M O A_{\log }$. Then

$$
\begin{aligned}
\frac{1}{|I|} \iint_{S(I)} & \left|H_{a}(f)^{\prime}(z)\right|^{2}\left(1-|z|^{2}\right) d m(z) \\
& \leq 2\left|g\left(z_{I}\right)\right|^{2} \frac{1}{|I|} \iint_{S(I)}\left|b^{\prime}(z)\right|^{2}\left(1-|z|^{2}\right) d m(z)+\frac{c}{\log \frac{4 \pi}{|I|}}\|b\|_{* *}^{2}\|g\|_{*}^{2} \\
& \leq c\|g\|_{*}^{2} \frac{\log ^{2} \frac{4 \pi}{|I|}}{|I|} \iint_{S(I)}\left|b^{\prime}(z)\right|^{2}\left(1-|z|^{2}\right) d m(z)+\frac{c}{\log \frac{4 \pi}{|I|}\|b\|_{* *}^{2}\|g\|_{*}^{2} .}
\end{aligned}
$$

Therefore,

$$
\lim _{|I| \rightarrow 0+} \frac{1}{|I|} \iint_{S(I)}\left|H_{a}(f)^{\prime}(z)\right|^{2}\left(1-|z|^{2}\right) d m(z)=0
$$

and $H_{a}(f) \in V M O A$.

For the converse, let $b \notin V M O A_{\log }$. Then there is some $\delta>0$ and some sequence $\left(I_{n}\right)$ of arcs such that $\left|I_{n}\right| \rightarrow 0$ and

$$
\frac{\log ^{2} \frac{4 \pi}{\left|I_{n}\right|}}{\left|I_{n}\right|} \iint_{S\left(I_{n}\right)}\left|b^{\prime}(z)\right|^{2}\left(1-|z|^{2}\right) d m(z) \geq \delta
$$

for all $n$. The previous estimates imply that

$$
\frac{1}{\left|I_{n}\right|} \iint_{S\left(I_{n}\right)}\left|H_{a}(f)^{\prime}(z)\right|^{2}\left(1-|z|^{2}\right) d m(z) \geq \frac{\delta}{2} \frac{\left|g\left(z_{I_{n}}\right)\right|^{2}}{\log ^{2} \frac{4 \pi}{\left|I_{n}\right|}}-\frac{c}{\log \frac{4 \pi}{\left|I_{n}\right|}}\|b\|_{* *}^{2}\|g\|_{*}^{2} .
$$

We shall construct some $g \in B M O A$ such that

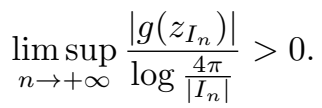

This will imply that

$$
\limsup _{n \rightarrow+\infty} \frac{1}{\left|I_{n}\right|} \iint_{S\left(I_{n}\right)}\left|H_{a}(f)^{\prime}(z)\right|^{2} d m(z)>0
$$

and, hence, that $H_{a}(f) \notin V M O A$.

\section{The construction of $\boldsymbol{g}$.}

Taking a subsequence, we may assume that $I_{n}$ accumulates to some point of $\mathbb{T}$. For simplicity of the formulas we shall replace $\mathbb{D}$ by the upper halfplane $\mathbb{H}=\{z=x+i y: y>0\}$ and $\mathbb{T}$ by the real line $\mathbb{R}$ and assume that the intervals $I_{n}$ of $\mathbb{R}$ accumulate to 0 .

Let $z_{n}=z_{I_{n}}=x_{n}+i y_{n}$, where $y_{n}=\left|I_{n}\right| \rightarrow 0$ and $x_{n}(\rightarrow 0)$ is the midpoint of $I_{n}$. 
We consider a fixed function $\phi:(0,+\infty) \rightarrow[0,1]$ with the following properties:

(i) $\phi(x)=0$ for $x \geq 2$,

(ii) $\phi(x)=1$ for $0<x \leq 1$,

(iii) $\phi$ is smooth in $(0,+\infty)$.

We then extend $\phi:(-\infty, 0) \cup(0,+\infty) \rightarrow[-1,1]$ so that

(iv) $\phi$ is odd.

We also consider the Hilbert transform

$$
H \phi(x)=\mathrm{p} \cdot \mathrm{v} \cdot \frac{1}{\pi} \int_{\mathbb{R}} \frac{\phi(t)}{x-t} d t
$$

and the analytic function

$$
(\phi+i H \phi)(z)=\frac{1}{\pi i} \int_{\mathbb{R}} \frac{\phi(t)}{z-t} d t,
$$

where $z \in \mathbb{H}$. Now it is easy to prove that

$$
|(\phi+i H \phi)(z)| \leq \frac{4}{|z|^{2}}, \quad z \in \mathbb{H},|z| \geq 3
$$

and

$$
|(\phi+i H \phi)(z)| \geq \log \frac{1}{|z|}, \quad z \in \mathbb{H},|z| \leq \delta
$$

for some appropriate fixed $\delta$ such that $0<\delta \leq \frac{1}{2}$. For the first, we assume $|z| \geq 3$ and find

$$
\begin{aligned}
|(\phi+i H \phi)(z)| & =\frac{1}{\pi}\left|\int_{\mathbb{R}} \frac{\phi(t)}{z-t} d t\right|=\frac{1}{\pi}\left|\int_{\mathbb{R}}\left(\frac{1}{z-t}-\frac{1}{z}\right) \phi(t) d t\right| \\
& =\frac{1}{\pi}\left|\int_{-2}^{2} \frac{t}{z(z-t)} \phi(t) d t\right| \leq \frac{6}{\pi|z|^{2}} \int_{0}^{2} t \phi(t) d t \leq \frac{4}{|z|^{2}} .
\end{aligned}
$$

For the second, we assume that $|z| \leq \frac{1}{2}$ and find

$$
\begin{aligned}
|(\phi+i H \phi)(z)| & \geq \frac{1}{\pi}\left|\int_{0}^{1} \frac{1}{z-t} d t-\int_{-1}^{0} \frac{1}{z-t} d t\right|-\frac{1}{\pi}\left|\int_{1 \leq|t| \leq 2} \frac{\phi(t)}{z-t} d t\right| \\
& \geq 2 \log \frac{1}{|z|}-c .
\end{aligned}
$$

First case: Suppose there is some $c>0$ such that

$$
\left|x_{n}\right| \leq c \sqrt{y_{n}}
$$

for all $n$. Of course, then $\left|z_{n}\right| \leq c \sqrt{y_{n}}=c \sqrt{\left|I_{n}\right|}$ for all $n$. 
Let $g=\phi+i H \phi$. Then $g \in B M O A$, since $\phi \in L^{\infty}$. For large $n$,

$$
\left|g\left(z_{n}\right)\right| \geq \log \frac{1}{\left|z_{n}\right|} \geq \log \frac{1}{c \sqrt{\left|I_{n}\right|}}
$$

and thus

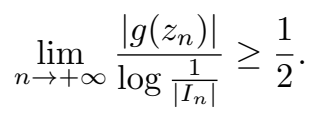

Second case: Suppose that $\lim _{\sup _{n \rightarrow+\infty}} \frac{\left|x_{n}\right|}{\sqrt{y_{n}}}=+\infty$ and, taking a subsequence, we may assume that

$$
\lim _{n \rightarrow+\infty} \frac{\left|x_{n}\right|}{\sqrt{y_{n}}}=+\infty .
$$

Now, let

$$
\phi_{n}(x)=\phi\left(\frac{x-x_{n}}{\sqrt{y_{n}}}\right) .
$$

Then $\phi_{n}$ is supported in the interval $\left[x_{n}-2 \sqrt{y_{n}}, x_{n}+2 \sqrt{y_{n}}\right]$ and, taking a further subsequence, we may assume that these intervals are pairwise disjoint. This implies that the function

$$
\psi=\sum_{k=1}^{+\infty} \phi_{k}
$$

is in $L^{\infty}$ and, more precisely, $|\psi| \leq 1$ a.e. in $\mathbb{R}$. Finally, we define

$$
g(z)=(\psi+i H \psi)(z)=\sum_{k=1}^{+\infty}(\phi+i H \phi)\left(\frac{z-x_{k}}{\sqrt{y_{k}}}\right), \quad z \in \mathbb{H} .
$$

Since $\psi \in L^{\infty}$, we have that $g \in B M O A$. Using one of the previous estimates, we see that for large $n$

$$
\left|(\phi+i H \phi)\left(\frac{z_{n}-x_{n}}{\sqrt{y_{n}}}\right)\right|=\left|(\phi+i H \phi)\left(i \sqrt{y_{n}}\right)\right| \geq \log \frac{1}{\sqrt{y_{n}}}=\frac{1}{2} \log \frac{1}{\left|I_{n}\right|} .
$$

We may now assume that $\left|x_{n+1}\right| \leq \frac{1}{2}\left|x_{n}\right|$ and that $y_{n+1} \leq y_{n}$ for all $n$. Then for $k<n$ we have $\left|\frac{x_{n}-x_{k}+i y_{n}}{\sqrt{y_{k}}}\right| \geq\left|\frac{x_{n}-x_{k}}{\sqrt{y_{k}}}\right| \geq \frac{\left|x_{k}\right|}{2 \sqrt{y_{k}}} \geq 3$ and for $k>n$ we have $\left|\frac{x_{n}-x_{k}+i y_{n}}{\sqrt{y_{k}}}\right| \geq\left|\frac{x_{n}-x_{k}}{\sqrt{y_{k}}}\right| \geq \frac{\left|x_{n}\right|}{2 \sqrt{y_{n}}} \geq 3$. By our estimates,

$$
\sum_{k=1}^{n-1}\left|(\phi+i H \phi)\left(\frac{z_{n}-x_{k}}{\sqrt{y_{k}}}\right)\right| \leq 4 \sum_{k=1}^{n-1} \frac{y_{k}}{\left(x_{n}-x_{k}\right)^{2}+y_{n}^{2}} \leq 16 \sum_{k=1}^{n-1} \frac{y_{k}}{x_{k}^{2}}
$$


and

$$
\sum_{k=n+1}^{+\infty}\left|(\phi+i H \phi)\left(\frac{z_{n}-x_{k}}{\sqrt{y_{k}}}\right)\right| \leq 4 \sum_{k=n+1}^{+\infty} \frac{y_{k}}{\left(x_{n}-x_{k}\right)^{2}+y_{n}^{2}} \leq 4 \sum_{k=n+1}^{+\infty} \frac{y_{k}}{x_{k}^{2}} .
$$

Since we may choose the intervals so that $\frac{\left|x_{n}\right|}{\sqrt{y_{n}}} \rightarrow+\infty$ fast enough, we may suppose that

$$
m=\sum_{k=1}^{+\infty} \frac{y_{k}}{x_{k}^{2}}<+\infty
$$

Therefore

$$
\left|g\left(z_{n}\right)\right| \geq \frac{1}{2} \log \frac{1}{\left|I_{n}\right|}-16 m
$$

and, finally,

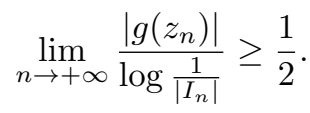

\section{References}

[1] O. Blasco, M. D. Contreras, S. Díaz-Madrigal, J. Martínez, M. Papadimitrakis, and A. G. Siskakis, Semigroups of composition operators and integral operators in spaces of analytic functions, Ann. Acad. Sci. Fenn. Math. 38(1) (2013), 67-89. DOI: 10.5186/aasfm. 2013.3806.

[2] J. Cima and D. A. Stegenga, Hankel operators on $H^{p}$, in: "Analysis at Urbana", Vol. I (Urbana, IL, 1986-1987), London Math. Soc. Lecture Note Ser. 137, Cambridge Univ. Press, Cambridge, 1989, pp. $133-150$.

[3] S. Janson, J. Peetre, and S. Semmes, On the action of Hankel and Toeplitz operators on some function spaces, Duke Math. J. 51(4) (1984), 937-958. DOI: 10.1215/S0012-7094-84-05142-1.

[4] M. Papadimitrakis and J. A. Virtanen, Hankel and Toeplitz transforms on $H^{1}$ : continuity, compactness and Fredholm properties, Integral Equations Operator Theory 61(4) (2008), 573-591. DOI: 10.1007/s00020-008-1609-2.

[5] V. A. Tolokonnikov, Hankel and Toeplitz operators in Hardy spaces, (Russian), Investigations on linear operators and the theory of functions, XIV, Zap. Nauchn. Sem. Leningrad. Otdel. Mat. Inst. Steklov. (LOMI) 141 (1985), 165-175, 191. 
Department of Mathematics

University of Crete

Knossos Ave.

71409 Iraklio

Greece

E-mail address: papadim@math.uoc.gr

Rebut el 5 de desembre de 2012. 